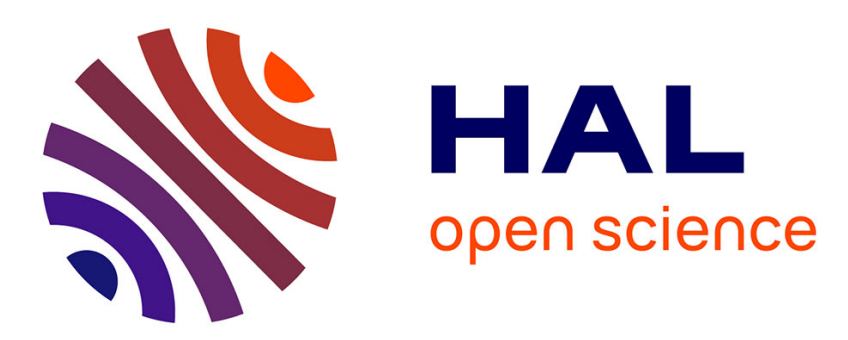

\title{
Freezing and low temperature entropy: the case of mean-field Gaussian model
}

Flora Koukiou

\section{To cite this version:}

Flora Koukiou. Freezing and low temperature entropy: the case of mean-field Gaussian model. Journal of Mathematical Physics, 2020, 10.1063/1.5063727 . hal-01870790v2

\section{HAL Id: hal-01870790 \\ https://hal.science/hal-01870790v2}

Submitted on 29 Aug 2020

HAL is a multi-disciplinary open access archive for the deposit and dissemination of scientific research documents, whether they are published or not. The documents may come from teaching and research institutions in France or abroad, or from public or private research centers.
L'archive ouverte pluridisciplinaire HAL, est destinée au dépôt et à la diffusion de documents scientifiques de niveau recherche, publiés ou non, émanant des établissements d'enseignement et de recherche français ou étrangers, des laboratoires publics ou privés. 


\title{
Freezing and low temperature entropy I : the case of mean-field Gaussian model
}

\author{
Flora KOUKIOU \\ CY Cergy Paris Université \\ Laboratoire de physique théorique et modélisation (CNRS UMR 8089) \\ F-95302 Cergy-Pontoise \\ flora.koukiou@cyu.fr
}

\begin{abstract}
The freezing phenomenon is studied in connection with the low temperature behaviour of the entropy of the Gibbs measure. In particular, in the case of the mean-field Gaussian spin glass model, from the study of a functional relation between the free energies at two different temperatures, we show that the maximum value of the inverse temperature beyond which the specific entropy of the Gibbs measure cannot be strictly positive is given by $\beta_{*}=4 \log 2$. The low temperature entropy of the Random Energy Model is also discussed.
\end{abstract}

\section{Introduction and main result}

During the last decades, the behaviour of random Gibbs measures associated with log-correlated random distributions has been extensively studied by the physics and mathematics communities. Initialy addressed in the case of random polymers [7] on trees, it has recently attracted considerable interest in the context of extreme value statistics and the Laplace functional of the randomly shifted decorated Poisson point processes (SDPPP) [18, 15]. The non-trivial behaviour of these measures at low temperatures (essentially dominated by the local extreme values of the associated random fields) gives rise to the freezing phenomenon. Freezing can be also characterized from the behaviour of the free energy at low temperatures [10, 4, 15] or from the properties of multifractal spectrum of the random measure [9].

An interesting question is whether freezing is related with the entropy of the measure. Indeed, it can be proved that freezing can be equally related with the vanishing of the entropy of the corresponding Gibbs measures in the case of random polymers on trees, multiplicative chaos and mean-field spin-glass models. Namely, for these modes, there exists a freezing temperature beyond which the specific entropy of the Gibbs measure cannot be strictly positive. This will be given in [14].

The purpose of this note is to present in detail the relationship between entropy and freezing in the case of the (widely studied) mean-field Gaussian spin-glass model. In particular, we introduce a simple, yet rigorous, method allowing the estimation the value of the freezing temperature at which the mean entropy of the Gibbs measure vanishes. Our approach is totally self-contained; we solely make use of the existence of the 
thermodynamic limit of the quenched specific free energy [12] and its self-averaging property [17]. Notice that the convexity of the free energy w.r.t. the inverse temperature implies its sub-differentiability which is sufficient to prove some of our results (in particular the lower bound of the free energy). Nevertheless, as we don't use here the Parisi measure — which is indeed differentiable [19, 16] - , we shall assume in this work the differentiability of the free energy to simplify and complete the proof.

We recall first some basic definitions. Suppose that a finite set of $n$ sites is given. With each site we associate the one-spin space $\Sigma:=\{1,-1\}$. The natural configuration space is then the product space $\Sigma_{n}=\Sigma^{n}=\{-1,1\}^{n}$, of card $\Sigma_{n}=2^{n}$. For each $\sigma \in \Sigma_{n}$, the finite volume Hamiltonian of the model is given by the following real-valued function on $\Sigma_{n}$

$$
H_{n}(\sigma, J)=-\frac{1}{\sqrt{n}} \sum_{1 \leq i<j \leq n} J_{i j} \sigma_{i} \sigma_{j},
$$

where the couplings $J=\left(J_{i j}\right)_{1 \leq i<j \leq n}$ are independent centered Gaussian variables of variance one.

At the inverse temperature $\beta=\frac{1}{T}>0$ (we take units such that the Boltzmann constant $k=1)$, the disorder-dependent partition function $Z_{n}(\beta, J)$, is given by the sum of the Boltzmann factors

$$
Z_{n}(\beta, J)=\sum_{\sigma \in \Sigma_{n}} e^{-\beta H_{n}(\sigma, J)} .
$$

Moreover, if $E_{J}$ denotes the expectation with respect to the randomness $J$, it is very simple to show that $E_{J} Z_{n}(\beta, J)=2^{n} e^{\frac{\beta^{2}}{4}(n-1)}$.

For fixed randomness, the corresponding Gibbs probability measure is denoted by $\mu_{n, \beta, J}(\sigma)$ and given by:

$$
\mu_{n, \beta}(\sigma)=\frac{e^{-\beta H_{n}(\sigma, J)}}{Z_{n}(\beta, J)} .
$$

We recall also the definition of the entropy of $\mu_{n, \beta}(\sigma): S\left(\mu_{n, \beta}\right)=-\sum_{\sigma} \mu_{n, \beta}(\sigma) \log \mu_{n, \beta}(\sigma)$.

The real functions

$$
f_{n}(\beta)=\frac{1}{n} E_{J} \log Z_{n}(\beta, J)
$$

and

$$
\bar{f}_{n}(\beta)=\frac{1}{n} \log E_{J} Z_{n}(\beta, J),
$$

define the quenched average of the specific free energy and the annealed specific free energy respectively. We denote by $\bar{f}_{\infty}(\beta), f_{\infty}(\beta)^{1}$, the corresponding thermodynamic limits

$$
\bar{f}_{\infty}(\beta)=\lim _{n \rightarrow \infty} \bar{f}_{n}(\beta, J) \text { and } f_{\infty}(\beta)=\lim _{n \rightarrow \infty} f_{n}(\beta, J) .
$$

We define the critical, $\beta_{c}$, and the freezing, $\beta_{f}$, temperatures by

$$
\begin{gathered}
\beta_{c}=\sup \left\{\beta: f_{\infty}(\beta)=\bar{f}_{\infty}(\beta)\right\}, \\
\beta_{f}=\inf \left\{\beta \geq \beta_{c}: \lim _{n \rightarrow \infty} \frac{1}{n} S\left(\mu_{n, \beta_{f r}}\right)=0, \text { a.s. }\right\} .
\end{gathered}
$$

\footnotetext{
${ }^{1}$ These functions are related to the usual thermodynamic specific free energies $F_{\infty}$ and $\bar{F}_{\infty}$ through $f_{\infty}(\beta)=-\beta F_{\infty}(\beta)$ and $\bar{f}_{\infty}(\beta)=-\beta \bar{F}_{\infty}(\beta)$.
} 
In the previous setting, the reader can recognize the mean field spin glass SherringtonKirkpatrick (SK) model. Various other mean-field models (REM, polymers on trees, Gaussian multiplicative chaos, etc.) can be defined similarly. The SK model has been widely studied in the mathematics and physics literature and has led to the development of numerous important analytical techniques. Namely, after the first result on the self-averaging [17], the long-standing problem of the existence and uniqueness of the infinite volume limit of the free energy at low temperature $(\beta>1)$ is obtained by the use of interpolation techniques in [12]. A subsequent work [2] provided a broad variational principle over random overlap structures and various bounds on the free energy. Important mathematical arguments towards a rigorous proof of the original solution proposed by Parisi are obtained in [19] and have been completed by the proof that the Parisi measure at zero temperature is supported by infinitely many points [3].

On the other hand, the behaviour of the entropy of the Gibbs measure in the low temperature region remains poorly understood. For mean-field models the specific entropy decreases with the temperature and it can easily be estimated for the high temperature region. By lowering the temperature the entropy should eventually vanish [17] and an early result, given in [1], corroborates the idea that the entropy does not vanish very fast.

The main motivation of the present work is the link between freezing and entropy and is summarized in the following

Theorem: Assuming the differentiability of the free energy with respect to the inverse temperature, the freezing temperature of the Gaussian mean-field spin-glass model cannot exceed $\beta_{*}=4 \log 2=2.77258 \ldots$. Moreover, for the mean entropy $s\left(\mu_{\beta}\right)$ of the Gibbs measure, we have that

$$
\forall \beta \geq \beta_{*}, s\left(\mu_{\beta}\right)=s\left(\mu_{\beta_{*}}\right):=\lim _{n \rightarrow \infty} \frac{1}{n} S\left(\mu_{n, \beta_{*}}\right)=-\lim _{n \rightarrow \infty} \frac{1}{n} \sum_{\sigma} \mu_{n, \beta_{*}}(\sigma) \log \mu_{n, \beta_{*}}(\sigma)=0 .
$$

The formulation of the above theorem assumes that $\lim _{n \rightarrow \infty} \frac{1}{n} S\left(\mu_{n, \beta_{*}, J}\right)$ exists and is independent of $J$. This follows from general principles and can immediately be obtained from the existence and self-averaging of the low temperature specific free energy [12].

\section{Proof of the theorem}

\section{Preliminaries and notation}

We first recall that, for all $\beta>0$, the quenched limit $f_{\infty}(\beta)$ exists and is a convex function of $\beta\left[12\right.$. Let $\beta_{1} \equiv 1$. From the high temperature results [1], we have, almost surely, that

$$
\begin{aligned}
f_{\infty}\left(\beta_{1}\right) & =\lim _{n \rightarrow \infty} \frac{1}{n} E_{J} \log Z_{n}\left(\beta_{1}, J\right)=\lim _{n \rightarrow \infty} \frac{1}{n} \log E_{J} Z_{n}\left(\beta_{1}, J\right) \\
& =\bar{f}_{\infty}\left(\beta_{1}\right)=\log 2+\frac{\beta_{1}^{2}}{4}\left(=\log 2+\frac{1}{4}\right) .
\end{aligned}
$$


Thus, for $\beta=\beta_{1}$, the quenched limit $f_{\infty}\left(\beta_{1}\right)$ equals to the annealed one $\bar{f}_{\infty}\left(\beta_{1}\right)=\beta_{1}^{2} / 4+$ $\log 2$, where the term $\beta_{1}^{2} / 4$ comes from the mean value of the Boltzmann factor, i.e. the typical behaviour and the mean behaviour coincide at this temperature.

Considering now the graph of the annealed limit $\bar{f}_{\infty}(\beta)=\frac{\beta^{2}}{4}+\log 2$ and the straight line $\frac{\beta}{\beta_{1}} f_{\infty}\left(\beta_{1}\right)$ we can easily check that the two graphs intersect at $\beta_{1} \equiv 1$ and $\beta_{*} \equiv$ $4 \log 2=2,77258 \ldots$.

Moreover, at $\beta=\beta_{*}$, the annealed limit $\bar{f}_{\infty}\left(\beta_{*}\right)$, is simply related to the limit $f_{\infty}\left(\beta_{1}\right)$ by

$$
\bar{f}_{\infty}\left(\beta_{*}\right)=\frac{\beta_{*}^{2}}{4}+\log 2=\frac{\beta_{*}}{\beta_{1}}\left(\frac{\beta_{*} \beta_{1}}{4} \frac{\beta_{1}}{\beta_{*}} \log 2\right)=\frac{\beta_{*}}{\beta_{1}}\left(\log 2+\frac{1}{4}\right)=\frac{\beta_{*}}{\beta_{1}} f_{\infty}\left(\beta_{1}\right) .
$$

This simple - at first glance - functional relationship has motivated the present work. Indeed, from the link between the limits $f_{\infty}\left(\beta_{*}\right)$ and $f_{\infty}\left(\beta_{1}\right)$, it is intuitively appealing to expect an underlying relationship between the Gibbs measures at $\beta_{1}=1$ and $\beta_{*}=4 \log 2$. As we shall see later, this connection is provided by the relative entropy of $\mu_{\beta_{*}}$ w.r.t. $\mu_{\beta_{1}}$. It should be noticed that the value $\beta_{1}$ is crucial since the behaviour of the the free energy and the entropy are known at $\beta_{1}=1$.

With what preceedes in hand, one can define for all $\beta \geq 0$, the affine mapping $T_{\beta}$ : $\mathbb{R}_{+} \rightarrow \mathbb{R}_{+}$, by $T_{\beta} x=\frac{\beta}{\beta_{1}} x$; for $\beta=\beta_{*}$, this mapping reads on the values of the limits

$$
T_{\beta_{*}}: f_{\infty}\left(\beta_{1}\right) \mapsto \bar{f}_{\infty}\left(\beta_{*}\right)=\frac{\beta_{*}}{\beta_{1}} f_{\infty}\left(\beta_{1}\right)
$$

It is worth noting that the temperature $\beta_{1}$ is introduced in order to preserve the homogeneity of the formulæ. Nevertheless, not to be pedantic, the explicit dependence on it will be often dropped in the sequel. Let moreover denote by $a$ the deviation of the limit $f_{\infty}\left(\beta_{*}\right)$ from its annealed value:

$$
a:=\bar{f}_{\infty}\left(\beta_{*}\right)-f_{\infty}\left(\beta_{*}\right)=\frac{\beta_{*}}{\beta_{1}} f_{\infty}\left(\beta_{1}\right)-f_{\infty}\left(\beta_{*}\right) .
$$

We now introduce a slightly different notation which simplifies the proof. Let $W_{n}\left(\sigma, \beta_{1}\right)$ be the random weight defined by $W_{n}\left(\sigma, \beta_{1}\right)=e^{-\beta_{1} H_{n}(\sigma, J)} / e^{\frac{\beta_{1}}{2} n}$. For all $\beta \geq \beta_{1}$, we denote by $\bar{g}(\beta)$ and $g_{\infty}(\beta)$ the tilted annealed and quenched limits respectively,

$$
\begin{aligned}
& \bar{g}_{\infty}(\beta)=\lim _{n \rightarrow \infty} \frac{1}{n} \log E_{J} \sum_{\sigma} W_{n}^{\frac{\beta}{\beta_{1}}}\left(\sigma, \beta_{1}\right)=\bar{f}_{\infty}(\beta)-\frac{\beta}{2}, \\
& g_{\infty}(\beta)=\lim _{n \rightarrow \infty} \frac{1}{n} E_{J} \log \sum_{\sigma} W_{n}^{\frac{\beta}{\beta_{1}}}\left(\sigma, \beta_{1}\right)=f_{\infty}(\beta)-\frac{\beta}{2} .
\end{aligned}
$$

At the inverse temperatures $\beta_{1}$ and $\beta_{*}$ the quenched free energy simply reads

$$
g_{\infty}\left(\beta_{1}\right)=f_{\infty}\left(\beta_{1}\right)-\frac{\beta_{1}}{2}=\log 2+\frac{\beta_{1}^{2}}{4}-\frac{\beta_{1}}{2}=\log 2-\frac{1}{4}
$$

and,

$$
g_{\infty}\left(\beta_{*}\right)=f_{\infty}\left(\beta_{*}\right)-\frac{\beta_{*}}{2} .
$$




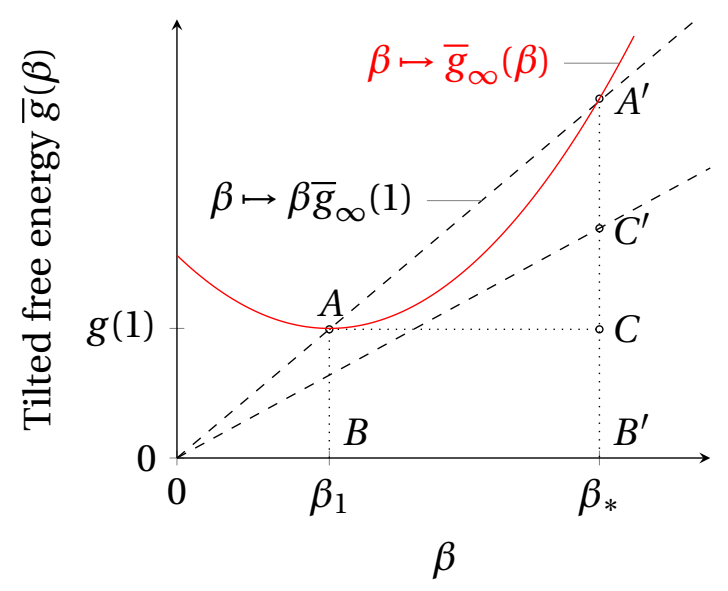

Figure 1: The graph $\beta \mapsto \bar{g}_{\infty}(\beta)$ depicts the annealed tilted free energy. The temperature $\beta_{*}$ is defined as the unique $\beta>1$ such that $\bar{g}_{\infty}(\beta)=\beta \bar{g}_{\infty}(1)$.

(The reader can recognize that the term $\frac{\beta}{2}$ we substract from the limit $g_{\infty}(\beta)$ corresponds to the lower convexity bound of the free energy).

In fig. 1, the graph of the convex function $\bar{g}_{\infty}(\beta)$ is plotted and it is easy to check that the minimum is reached at $\beta=1$. The same holds for the minimum of the limit $g_{\infty}(\beta)$ because $g_{\infty}$ is convex and $\bar{g}_{\infty}^{\prime}\left(\beta_{1}\right)=\bar{g}_{\infty}^{\prime}\left(\beta_{1}-0\right)=g_{\infty}^{\prime}\left(\beta_{1}-0\right)=0$.

With what precedes in mind, we can make two observations. First, at $\beta_{1}$, the entropy $s\left(\mu_{\beta_{1}}\right)$ equals $g_{\infty}\left(\beta_{1}\right)$, i.e.

$$
\begin{aligned}
s\left(\mu_{\beta_{1}}\right) & =\lim _{n \rightarrow \infty} \frac{1}{n} S\left(\mu_{n, \beta_{1}}\right)=-\lim _{n \rightarrow \infty} \frac{1}{n} \sum_{\sigma} \mu_{n, \beta_{1}}(\sigma) \log \mu_{n, \beta_{1}}(\sigma) \\
& =-\lim _{n \rightarrow \infty} \frac{1}{n} \sum_{\sigma} \mu_{n, \beta_{1}}(\sigma) \log e^{-\beta_{1} H_{n}(\sigma, J)}+f_{\infty}\left(\beta_{1}\right) \\
& =-\frac{\beta_{1}}{2}+f_{\infty}\left(\beta_{1}\right)=g_{\infty}\left(\beta_{1}\right)\left(=\log 2-\frac{1}{4}\right) .
\end{aligned}
$$

Second, we recall the definition of the relative entropy density $s\left(\lambda \mid \lambda^{\prime}\right)$ of the probability measure $\lambda$ w.r.t. the probability measure $\lambda^{\prime}$, defined on an arbitrary discrete space $X$ :

$$
s\left(\lambda \mid \lambda^{\prime}\right):= \begin{cases}\sum_{x \in X} \lambda(x) \log \frac{\lambda(x)}{\lambda^{\prime}(x)} & \text { if } \lambda \ll \lambda^{\prime} \\ +\infty & \text { otherwise }\end{cases}
$$

The relative entropy is a non-negative function, vanishing in case the two measures are equal, and gives the extent to which the measure $\lambda$ "differs" from the measure $\lambda^{\prime}$. Using this definition we can now relate the limit $g_{\infty}(\beta)$ with the relative entropy $s\left(\mu_{\beta_{1}} \mid \mu_{\beta}\right)$ for 
all $\beta \geq \beta_{1}$ : Namely,

$$
\begin{aligned}
s\left(\mu_{\beta_{1}} \mid \mu_{\beta}\right): & =\lim _{n \rightarrow \infty} \frac{1}{n} \sum_{\sigma} \mu_{n, \beta_{1}}(\sigma) \log \frac{\mu_{n, \beta_{1}}(\sigma)}{\mu_{n, \beta}(\sigma)} \\
& =-s\left(\mu_{\beta_{1}}\right)+\lim _{n \rightarrow \infty} \frac{\beta}{n} \sum_{\sigma} \mu_{n, \beta_{1}}(\sigma) H_{n}(\sigma, J)+\lim _{n \rightarrow \infty} \frac{1}{n} \log Z_{n}(\beta, J) \\
& =-s\left(\mu_{\beta_{1}}\right)-\beta f_{\infty}^{\prime}\left(\beta_{1}\right)+f_{\infty}(\beta) \\
& =-s\left(\mu_{\beta_{1}}\right)-\frac{\beta \beta_{1}}{2}+f_{\infty}(\beta)=-s\left(\mu_{\beta_{1}}\right)+g_{\infty}(\beta),
\end{aligned}
$$

(where we have used the fact $f_{\infty}^{\prime}\left(\beta_{1}\right)=-\lim _{n \rightarrow \infty} \sum_{\sigma} \mu_{n, \beta_{1}}(\sigma) H_{n}(\sigma, J)=\beta_{1} / 2=1 / 2$ ).

Thus, for all $\beta \geq \beta_{1}$, the tilted quenched limit $g_{\infty}(\beta)$ is simply given by

$$
g_{\infty}(\beta)=s\left(\mu_{\beta_{1}}\right)+s\left(\mu_{\beta_{1}} \mid \mu_{\beta}\right)=g_{\infty}\left(\beta_{1}\right)+s\left(\mu_{\beta_{1}} \mid \mu_{\beta}\right) .
$$

By making now use of the mapping $T_{\beta_{*}}$, one readily gets

$$
\bar{g}_{\infty}\left(\beta_{*}\right)=\frac{\beta_{*}}{\beta_{1}} g_{\infty}\left(\beta_{1}\right)=\frac{\beta_{*}}{\beta_{1}} s\left(\mu_{\beta_{1}}\right)=g_{\infty}\left(\beta_{*}\right)+a,
$$

and moreover, from eq 2 , we have $\mathrm{2}^{2}$ that for $\beta=\beta_{*}$,

$$
s\left(\mu_{\beta_{1}} \mid \mu_{\beta_{*}}\right)+a=\frac{\beta_{*}}{\beta_{1}} s\left(\mu_{\beta_{1}}\right)-s\left(\mu_{\beta_{1}}\right)=\left(\beta_{*}-1\right) s\left(\mu_{\beta_{1}}\right)=\left(\beta_{*}-1\right) g_{\infty}\left(\beta_{1}\right) .
$$

It is important to stress that although eq. 2 is valid $\forall \beta \geq \beta_{1}$, eq. 3 is valid only at $\beta=\beta_{*}$.

In fig. 1. the limits $g_{\infty}\left(\beta_{1}\right)$ and $\bar{g}_{\infty}\left(\beta_{*}\right)$ are given by the lengths of the segments $A B$ and $A^{\prime} B^{\prime}$ respectively. Furthermore, the segment $C A^{\prime}$ equals to the sum $s\left(\mu_{\beta_{1}} \mid \mu_{\beta_{*}}\right)+a$. It follows that the (unknown) value of $g_{\infty}\left(\beta_{*}\right)$ correspond to the length of a segment $B^{\prime} C^{\prime}$, where the point $C^{\prime}$ lies between $C$ and $A^{\prime}$.

We can now prove the theorem in two steps.

\section{Step 1: establishing a lower bound for the free energy}

Thanks to eqs. 3 and 3 we shall obtain a lower bound for the relative entropy $s\left(\mu_{\beta_{1}} \mid \mu_{\beta_{*}}\right)$ by comparing the limits $a$ and $g_{\infty}\left(\beta_{1}\right)\left(=s\left(\mu_{\beta_{1}}\right)\right)$ and by providing a geometric sketch of it. On one hand, we have that $\bar{g}_{\infty}\left(\beta_{*}\right)=\beta_{*} s\left(\mu_{\beta_{1}}\right)$ and $g_{\infty}\left(\beta_{*}\right)=s\left(\mu_{\beta_{1}}\right)+s\left(\mu_{\beta_{1}} \mid \mu_{\beta_{*}}\right)$, i.e. the limit $g_{\infty}\left(\beta_{*}\right)$ exceeds $s\left(\mu_{\beta_{1}}\right)$ by the relative entropy $s\left(\mu_{\beta_{1}} \mid \mu_{\beta_{*}}\right)$. Moreover, on the other hand, we have shown that $s\left(\mu_{\beta_{1}} \mid \mu_{\beta_{*}}\right)+a=\left(\beta_{*}-1\right) s\left(\mu_{\beta_{1}}\right)$. Suppose now that in fig. 1, the segment whose length equals $a$ is transported to some point $D$ below the point $C$ (with $D C^{\prime}=C A$ ) and since the lowest position of $D$ is when it coincides with $B^{\prime}$, it follows that $a$ cannot be bigger than $s\left(\mu_{\beta_{1}}\right)$ and $\left.s\left(\mu_{\beta_{1}} \mid \mu_{\beta_{*}}\right)\right) \geq\left(\beta_{*}-2\right) s\left(\mu_{\beta_{1}}\right)=C C^{\prime}$. In other words, $a \leq s\left(\mu_{\beta_{1}}\right)$, and,

$$
s\left(\mu_{\beta_{1}} \mid \mu_{\beta_{*}}\right) \geq\left(\beta_{*}-2\right) s\left(\mu_{\beta_{1}}\right) .
$$

This implies the following lower bound for the limit $g_{\infty}\left(\beta_{*}\right)$ :

$$
g_{\infty}\left(\beta_{*}\right) \geq s\left(\mu_{\beta_{1}}\right)+\left(\beta_{*}-2\right) s\left(\mu_{\beta_{1}}\right)=\left(\beta_{*}-1\right) s\left(\mu_{\beta_{1}}\right)=\left(\beta_{*}-1\right) g_{\infty}\left(\beta_{1}\right) .
$$

${ }^{2}$ It is recalled that $\left(\beta_{*}-1\right) s\left(\mu_{\beta_{1}}\right)$ means $\left(\frac{\beta_{*}}{\beta_{1}}-1\right) s\left(\mu_{\beta_{1}}\right)$. 


\section{Step 2: vanishing of the entropy at $\beta_{*}$}

In the second step of the proof we shall show that in case the previous lower bound is saturated, the entropy $s\left(\mu_{\beta_{*}}\right)$ vanishes. For this, we consider the relative entropy $s\left(\mu_{\beta_{*}} \mid \mu_{\beta_{1}}\right)$,

$$
\begin{aligned}
s\left(\mu_{\beta_{*}} \mid \mu_{\beta_{1}}\right): & =\lim _{n \rightarrow \infty} \frac{1}{n} \sum_{\sigma} \mu_{n, \beta_{*}}(\sigma) \log \frac{\mu_{n, \beta_{*}}(\sigma)}{\mu_{n, \beta_{1}}(\sigma)} \\
& =-s\left(\mu_{\beta_{*}}\right)-\lim _{n \rightarrow \infty} \frac{1}{n} \sum_{\sigma} \mu_{n, \beta_{*}}(\sigma) \log \mu_{n, \beta_{1}}(\sigma) \\
& =-s\left(\mu_{\beta_{*}}\right)-\sum_{\sigma} \mu_{n, \beta_{*}}(\sigma) \log e^{-\beta_{1} H_{n}(\sigma, J)}+f_{\infty}\left(\beta_{1}\right) \\
& =-s\left(\mu_{\beta_{*}}\right)+s\left(\mu_{\beta_{1}}\right)-A_{\infty}
\end{aligned}
$$

where

$$
A_{\infty}=\lim _{n \rightarrow \infty} \frac{1}{n}\left(\sum_{\sigma} \mu_{n, \beta_{*}}(\sigma) \log e^{-\beta_{1} H_{n}(\sigma, J)}-\sum_{\sigma} \mu_{n, \beta_{1}}(\sigma) \log e^{-\beta_{1} H_{n}(\sigma, J)}\right) .
$$

Moreover, since $\lim _{n \rightarrow \infty} \frac{\beta_{*}}{n} \sum_{\sigma} \mu_{n, \beta_{1}}(\sigma) \log e^{-\beta_{1} H_{n}(\sigma, J)}=\beta_{*} f_{\infty}^{\prime}\left(\beta_{1}\right)=\beta_{*} / 2$, we have that

$$
\begin{aligned}
g_{\infty}\left(\beta_{*}\right) & =s\left(\mu_{\beta_{*}}\right)+\lim _{n \rightarrow \infty} \frac{1}{n} \sum_{\sigma} \mu_{n, \beta_{*}}(\sigma) \log e^{-\beta_{*} H_{n}(\sigma, J)}-\frac{\beta_{*}}{2} \\
& =s\left(\mu_{\beta_{*}}\right)+\lim _{n \rightarrow \infty} \frac{1}{n}\left(\sum_{\sigma} \mu_{n, \beta_{*}}(\sigma) \log e^{-\beta_{*} H_{n}(\sigma, J)}-\sum_{\sigma} \mu_{n, \beta_{1}}(\sigma) \log e^{-\beta_{*} H_{n}(\sigma, J)}\right) \\
& =s\left(\mu_{\beta_{*}}\right)+\beta_{*} A_{\infty}, \\
& =s\left(\mu_{\beta_{1}}\right)+\left(\beta_{*}-1\right) A_{\infty}-s\left(\mu_{\beta_{*}} \mid \mu_{\beta_{1}}\right) .
\end{aligned}
$$

Recalling now that $g_{\infty}\left(\beta_{*}\right)=s\left(\mu_{\beta_{1}}\right)+s\left(\mu_{\beta_{1}} \mid \mu_{\beta_{*}}\right)$, we obtain the following equation valid only at $\beta_{*}$ - between the two relative entropies

$$
s\left(\mu_{\beta_{*}} \mid \mu_{\beta_{1}}\right)+s\left(\mu_{\beta_{1}} \mid \mu_{\beta_{*}}\right)=\left(\beta_{*}-1\right) A_{\infty} .
$$

We can now complete the proof by using the following argument. Assume that the obtained bound for the limit $a$ is saturated i.e. $a=a^{\max }=s\left(\mu_{\beta_{1}}\right)=s\left(\mu_{\beta_{*}}\right)+A_{\infty}+s\left(\mu_{\beta_{*}} \mid \mu_{\beta_{1}}\right)$. In this case, $s^{\min }\left(\mu_{\beta_{1}} \mid \mu_{\beta_{*}}\right)=\left(\beta_{*}-2\right) s\left(\mu_{\beta_{1}}\right)$, and $g_{\infty}^{\min }\left(\beta_{*}\right)=\left(\beta_{*}-1\right) s\left(\mu_{\beta_{1}}\right)$. From eq. 4 we obtain that

$$
a^{\max }=s\left(\mu_{\beta_{1}}\right)=\beta_{*} s\left(\mu_{\beta *} \mid \mu_{\beta_{1}}\right)+\left(\beta_{*}-1\right) s\left(\mu_{\beta_{*}}\right),
$$

and, moreover

$$
A_{\infty}=\left(\beta_{*}-1\right) s\left(\mu_{\beta_{*}} \mid \mu_{\beta_{1}}\right)+\left(\beta_{*}-2\right) s\left(\mu_{\beta_{*}}\right) .
$$

We notice that in case the entropy of the measure vanishes, i.e. $s\left(\mu_{\beta_{*}}\right)=0$ one has $a^{\max }=\beta_{*} s\left(\mu_{\beta_{*}} \mid \mu_{\beta_{1}}\right), A_{\infty}=\left(\beta_{*}-1\right) s\left(\mu_{\beta_{*}} \mid \mu_{\beta_{1}}\right)$ and $g_{\infty}^{\min }\left(\beta_{*}\right)=\beta_{*} A_{\infty}$.

To prove the converse, we remark that the limit $g_{\infty}^{\min }\left(\beta_{*}\right)$ can be pulled backwards at $\beta=\beta_{1}$ by the mapping $T_{\beta}$, namely

$$
T^{-1} g_{\infty}^{\min }\left(\beta_{*}\right)=\frac{\beta_{1}}{\beta_{*}} g_{\infty}^{\min }\left(\beta_{*}\right)=\frac{\beta_{1}}{\beta_{*}}\left(\beta_{*}-1\right) s\left(\mu_{\beta_{1}}\right) .
$$


Similarly,

$$
T^{-1} a^{\max }=\frac{\beta_{1}}{\beta_{*}} s\left(\mu_{\beta_{1}}\right)
$$

Since now

$$
\begin{aligned}
g_{\infty}^{\min }\left(\beta_{*}\right) & =\left(\beta_{*}-1\right) s\left(\mu_{\beta_{1}}\right) \\
& =\beta_{*} T^{-1} g_{\infty}^{\min }\left(\beta_{*}\right)=\beta_{*}\left(\frac{\beta_{1}}{\beta_{*}}\left(\beta_{*}-1\right) s\left(\mu_{\beta_{1}}\right)\right),
\end{aligned}
$$

we conclude that

$$
A_{\infty}=\beta_{1}\left(\beta_{*}-1\right) \frac{s\left(\mu_{\beta_{1}}\right)}{\beta_{*}}=\left(\beta_{*}-1\right) \frac{s\left(\mu_{\beta_{1}}\right)}{\beta_{*}}, \quad s\left(\mu_{\beta_{*}} \mid \mu_{\beta_{1}}\right)=\beta_{1} \frac{s\left(\mu_{\beta_{1}}\right)}{\beta_{*}}=\frac{s\left(\mu_{\beta_{1}}\right)}{\beta_{*}} .
$$

Recalling that $s\left(\mu_{\beta_{1}}\right)=s\left(\mu_{\beta_{*}}\right)+A_{\infty}+s\left(\mu_{\beta_{*}} \mid \mu_{\beta_{1}}\right)$, we obtain that

$$
s\left(\mu_{\beta_{*}}\right)=0 .
$$

We have thus established that, under the assumption of saturation of the lower bound for $g_{\infty}\left(\beta_{*}\right)$, the entropy $s\left(\mu_{\beta_{*}}\right)$ vanishes and, moreover, $a=\beta_{*} s\left(\mu_{\beta_{*}} \mid \mu_{\beta_{1}}\right)$. Thus, the sum $s\left(\mu_{\beta_{*}} \mid \mu_{\beta_{1}}\right)+A_{\infty}$ reaches its maximum value, i.e. the maximum value of the difference $s\left(\mu_{\beta_{1}}\right)-s\left(\mu_{\beta_{*}}\right)$. One can now remark that, if $g_{\infty}\left(\beta_{*}\right)>g_{\infty}^{\min }\left(\beta_{*}\right)$, (i.e. the relative entropy $s\left(\mu_{\beta_{1}} \mid \mu_{\beta_{*}}\right)$ is bigger than its lower bound), the entropy $s\left(\mu_{\beta_{*}}\right)$ has already vanished at an inverse temperature smalle1 ${ }^{3}$ than $\beta_{*}$ since $\left(\beta_{*}-1\right) s\left(\mu_{\beta_{1}}\right) / \beta_{*} \leq A_{\infty} \leq s\left(\mu_{\beta_{1}}\right)$ and, contravariantly $0 \leq s\left(\mu_{\beta_{*}} \mid \mu_{\beta_{1}}\right) \leq s\left(\mu_{\beta_{1}}\right) / \beta_{*}$ so that their sum is always $s\left(\mu_{\beta_{1}}\right)$.

For $\beta>\beta_{f}$ the entropy remains zero and (since the entropy cannot be strictly positive for all values of $\beta>\beta_{*}$ ), the freezing temperature of the model is given by $\beta_{f} \leq \beta_{*}$. Thus, the value $\beta_{*}$ provides the maximum value beyond which the specific entropy of the Gibbs measure cannot be strictly positive.

Recalling the positivity of the entropy and the geometric fact that at each point on the graph of a convex function lies above its sub-differential, we obtain $\forall \beta \geq \beta_{*}$, the lower boundary of the limit $g_{\infty}(\beta)$, illustrated by the dotted line in the fig. 1 which improves an early result obtained in [13]. Using also the upper spherical bound of [6], we conclude that the quenched limit $g_{\infty}(\beta)$ lies inside the shaded area illustrated in figure2.

One could notice that the obtained lower bound for the tilted limit $g_{\infty}^{\min }\left(\beta_{*}\right)$,

$$
g_{\infty}\left(\beta_{*}\right)=s\left(\mu_{\beta_{1}}\right)+s\left(\mu_{\beta_{1}} \mid \mu_{\beta_{*}}\right) \geq g_{\infty}^{\min }\left(\beta_{*}\right)=\left(\beta_{*}-1\right) s\left(\mu_{\beta_{1}}\right)=\frac{\beta_{*}^{2}}{4}-\frac{\beta_{*}}{2}+\frac{\beta_{1}^{2}}{4},
$$

implies, for the quenched free energy $f_{\infty}\left(\beta_{*}\right)$ of the model,

$$
f_{\infty}\left(\beta_{*}\right)=g_{\infty}\left(\beta_{*}\right)+\frac{\beta_{*}}{2} \geq \frac{\beta_{*}^{2}}{4}+\frac{1}{4}=2.1718 \ldots
$$

By making now use of the raw spherical (upper) bound [6] one has that almost surely,

$$
2.1718 \ldots \leq f_{\infty}\left(\beta_{*}\right) \leq 2.2058 \ldots
$$

\footnotetext{
${ }^{3}$ As explained in the Appendix, this arises in particular in the case of the REM.
} 


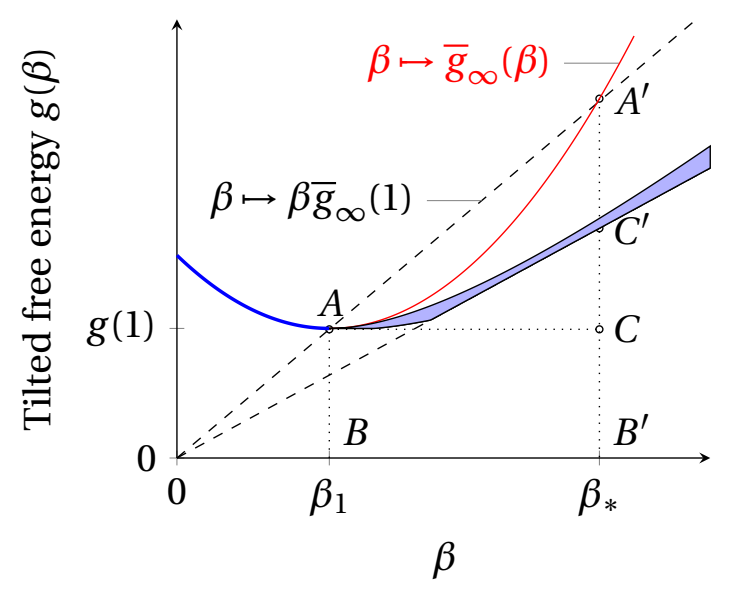

Figure 2: The graph $\beta \mapsto \bar{g}_{\infty}(\beta)$ depicts the annealed tilted free energy. The (low temperature) quenched function must lie within the shaded region, delimited from above by the spherical bound of [6] and below by the combination of the bound previously established in [13] and the lower bound obtained in the present work.

\section{Comments and concluding remarks}

In this note we proved that the mean entropy of the Gibbs measure is zero at the inverse (freezing) temperature $\beta_{*}=4 \log 2=2.7725 \ldots$. Obviously, the low temperature ultrametric picture occurs in the interval $\left[\beta_{1}, \beta_{f}\right]$ and is related to the Parisi solution.

The peculiar property arising at $\beta_{*}$ is the relationship between the deviation $a$ and the relative entropy (i.e. $a=\beta_{*} s\left(\mu_{\beta_{*}} \mid \mu_{\beta_{1}}\right)$ ). This relationship is observed in all meanfield models. In [14] we study the relation of of freezing with the entropy of the Gibbs measure for random polymers on trees [7] and the multiplicative chaos [5]. One can also readily check that $\beta_{*}=\beta_{c}^{2}$, where $\beta_{c}=2 \sqrt{\log 2}=1.6651 \ldots$ is the critical temperature of the Random Energy Model (REM). Both $\beta_{c}$ and $\beta_{*}$ are to be compared with the value at $\beta_{1} \equiv 1$, i.e. the maximum value of $\beta$ where the free energies of the two models coincide. What we learn by the comparison of the relative entropies of the two models is that the Gibbs measure of the Sherrington-Kirkpatrick has seemingly different concentration properties than for the REM. As recalled in the Appendix, in the case of the REM, the quenched and annealed limit of the free energy are equal for all temperatures of positive entropy and consequently the critical temperature coincides with the freezing one. This does not occur in the case of SK, where the two limits are equal only for $\beta \leq 1$. Rather surprisingly, one verifies that this lower bound can also be expressed by the free energy at the "dual" [11] temperature $1 / \beta_{c}$. In particular,

$$
f_{\infty}\left(\beta_{*}\right) \geq \beta_{*} f_{\infty}\left(1 / \beta_{c}\right),
$$

where $\beta_{c}$ is the critical temperature of the REM.

Another remark concerns the Hausdorff dimension of the support of the Gibbs measure. Using the theorem of this note one can show that this dimension vanishes at $\beta_{*}$ and thus relate the freezing transition with the behaviour of the multifractal spectrum introduced in [9]. 
We also mention two open questions of particular interest, namely the pertinence of our result to the low temperature behaviour of the Parisi measure and the precise connection of the duality [11] with the freezing transition.

Note also that, although evident, the non-standard expression of the free energy in terms of the entropy $s\left(\mu_{\beta_{1}}\right)$ and the relative entropy $s\left(\mu_{\beta_{1}} \mid \mu_{\beta}\right)$ (eq. 2$), \forall \beta>\beta_{1}$, has special importance not only for the present proof. Indeed, one can apply this expression to recover information about the measure at $\beta_{1}$, from information represented by $s\left(\mu_{\beta_{1}} \mid \mu_{\beta}\right)$ (for instance, in the case of compressed sensing in signal processing).

\section{References}

[1] M. Aizenman, J. L. Lebowitz, and D. Ruelle. Some rigorous results on the Sherrington-Kirkpatrick spin glass model. Comm. Math. Phys., 112(1):3-20, 1987.

[2] Michael Aizenman, Robert Sims, and Shannon L. Starr. Extended variational principle for the Sherrington-Kirkpatrick spinglass model. Phys. Rev. B, 68:214403, Dec 2003.

[3] Antonio Auffinger, Wei-Kuo Chen, and Qiang Zeng. The SK model is infinite step replica symmetry breaking at zero temperature. Comm. Pure Appl. Math., 73(5):921-943, 2020.

[4] David Carpentier and Pierre Le Doussal. Glass transition of a particle in a random potential, front selection in nonlinear renormalization group, and entropic phenomena in liouville and sinh-gordon models. Phys. Rev. E, 63:026110, Jan 2001.

[5] P. Collet and F. Koukiou. Large deviations for multiplicative chaos. Comm. Math. Phys., 147(2):329-342, 1992.

[6] F. Comets. A spherical bound for the Sherrington-Kirkpatrick model. Astérisque, 236:103-108, 1996. Hommage à P. A. Meyer et J. Neveu.

[7] B. Derrida and H. Spohn. Polymers on disordered trees, spin glasses, and traveling waves. J. Statist. Phys., 51(5-6):817-840, 1988. New directions in statistical mechanics (Santa Barbara, CA, 1987).

[8] Bernard Derrida. Random-energy model: an exactly solvable model of disordered systems. Phys. Rev. B (3), 24(5):2613-2626, 1981.

[9] Yan V. Fyodorov. Multifractality and freezing phenomena in random energy landscapes: An introduction. Physica A: Statistical Mechanics and its Applications, 389(20):4229-4254, Oct 2010.

[10] Yan V. Fyodorov and Jean-Philippe Bouchaud. Freezing and extreme-value statistics in a random energy model with logarithmically correlated potential. J. Phys. A, 41(37):372001, 12, 2008.

[11] Yan V. Fyodorov, Pierre Le Doussal, and Alberto Rosso. Statistical mechanics of logarithmic REM: duality, freezing and extreme value statistics of $1 / f$ noises generated by Gaussian free fields. J. Stat. Mech. Theory Exp., (10):P10005, 32, 2009.

[12] Francesco Guerra and Fabio Lucio Toninelli. The thermodynamic limit in mean field spin glass models. Comm. Math. Phys., $230(1): 71-79,2002$.

[13] F. Koukiou. The low-temperature free energy of the Sherrington-Kirkpatrick spin glass model. Europhys. Lett., 33(2):95-98, 1996.

[14] F. Koukiou. Freezing and low temperature entropy II. In preparation, 2020.

[15] Thomas Madaule, Rémi Rhodes, and Vincent Vargas. Glassy phase and freezing of log-correlated Gaussian potentials. Ann. Appl. Probab., 26(2):643-690, 2016.

[16] Dmitry Panchenko. On differentiability of the Parisi formula. Electron. Commun. Probab., 13:241-247, 2008.

[17] L. A. Pastur and M. V. Shcherbina. Absence of self-averaging of the order parameter in the Sherrington-Kirkpatrick model. J. Statist. Phys., 62(1-2):1-19, 1991.

[18] Eliran Subag and Ofer Zeitouni. Freezing and decorated Poisson point processes. Comm. Math. Phys., 337(1):55-92, 2015.

[19] Michel Talagrand. The Parisi formula. Ann. of Math. (2), 163(1):221-263, 2006 


\section{A Appendix: The REM example}

In the following we apply our approach in the case of the Random Energy Model (REM). The REM, introduced in [8], is defined by a family $\left(E_{i}\right)_{i=1, \ldots, 2^{n}}$ of random, independent, centerd, identically distributed Gaussian variables of variance $n / 2$, corresponding to the ad hoc energy levels of the model. The high temperature behaviour of the REM is the same as the SK model. It is well known that the REM undergoes a phase transition at the critical inverse temperature $\beta_{c}=2 \sqrt{\log 2}$ and the entropy of the Gibbs measure vanishes almost surely $\forall \beta \geq \beta_{c}$ i.e. the freezing temperature coincides with the critical one. We recall moreover

$$
f_{\infty}(\beta)= \begin{cases}\frac{\beta^{2}}{4}+\log 2 & \text { for } \beta \leq \beta_{c}, \\ \beta \sqrt{\log 2} & \text { for } \beta \geq \beta_{c} .\end{cases}
$$

One can readily see that the functional relationship (1) between the annealed limits $\bar{f}_{\infty}\left(\beta_{*}\right)$ and $f_{\infty}\left(\beta_{1}\right)$ is also valid in the case of the REM. In the figure 3 , we present the mappings for the REM tilded functions.

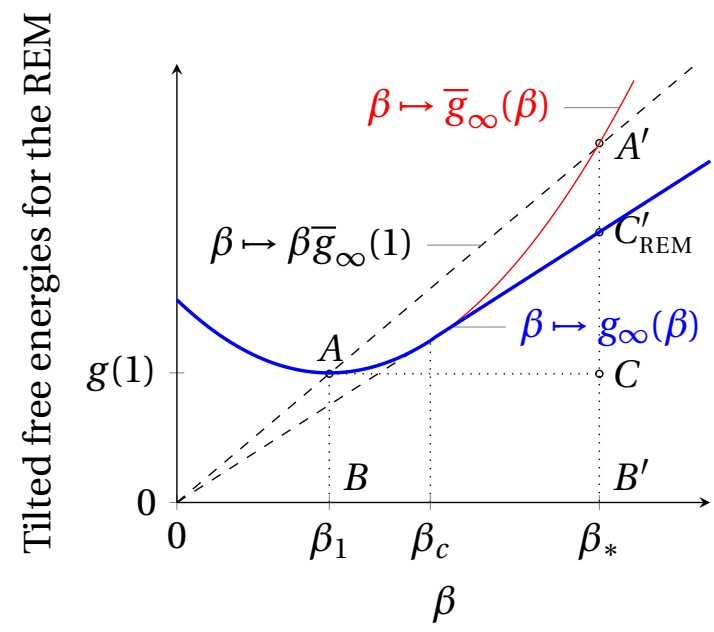

Figure 3: Mappings for the tilted functions of the REM.

Namely, the segment $C_{\mathrm{REM}}^{\prime} A^{\prime}$ corresponds to the difference $a^{\mathrm{REM}}$ and one can easily verify that

$$
a^{\mathrm{REM}}=\bar{g}_{\infty}\left(\beta_{*}\right)-g_{\infty}\left(\beta_{*}\right)=\frac{\beta_{*}^{2}}{4}+\log 2-\beta \sqrt{\log 2}=\log 2\left(\beta_{*}-2 \beta_{c}+1\right) .
$$


The relative entropy $s^{\mathrm{REM}}\left(\mu_{\beta_{*}} \mid \mu_{\beta_{1}}\right)$ is given by

$$
\begin{aligned}
s^{\mathrm{REM}}\left(\mu_{\beta_{*}} \mid \mu_{\beta_{1}}\right): & =\lim _{n \rightarrow \infty} \frac{1}{n} \sum_{\sigma} \mu_{n, \beta_{*}}(\sigma) \log \frac{\mu_{n, \beta_{*}}(\sigma)}{\mu_{n, \beta_{1}}(\sigma)} \\
& =-s\left(\mu_{\beta_{*}}\right)-\lim _{n \rightarrow \infty} \frac{1}{n} \sum_{\sigma} \mu_{n, \beta_{*}}(\sigma) \log \mu_{n, \beta_{1}}(\sigma) \\
& =s\left(\mu_{\beta_{1}}\right)-A_{\infty}^{\mathrm{REM}} \\
& =\frac{1}{4}\left(\beta_{*}-2 \beta_{c}+1\right)=\frac{a^{\mathrm{REM}}}{\beta_{*}} .
\end{aligned}
$$

As expected, the relative entropy $s^{\mathrm{REM}}\left(\mu_{\beta_{*}} \mid \mu_{\beta 1}\right)$ is smaller that the relative entropy we have obtained in the previous section; indeed the "difference" between the Gibbs measures at $\beta_{*}$ and $\beta_{1}$ is smaller in the case of REM than the SK. 